\title{
Effects of Sublingual Duster Mite Drops on Lung Function and Exhaled Nitric Oxide in Children with Multiple and Single Allergic Respiratory Diseases
}

\author{
Li Wang $\left(\mathbb{D}\right.$, Tao Ai $\mathbb{D}^{-}$, Ronghua Luo $\left(\mathbb{D}\right.$, Yinghong Fan $\left(\mathbb{D}\right.$, Huiling Liao $\mathbb{D}$, Wanmin Xia $\mathbb{D}^{\circ}$, \\ Cheng Xie $\mathbb{D}$, Yaping Duan $\mathbb{D}$, and Yanru Liu $\mathbb{1}$
}

Pediatric Respiratory Medicine Department, Chengdu Women's and Children's Central Hospital, School of Medicine, University of Electronic Science and Technology of China, Chengdu 611731, China

Correspondence should be addressed to Tao Ai; taoai2020@21cn.com

Received 17 June 2020; Accepted 20 October 2020; Published 4 November 2020

Academic Editor: Pierachille Santus

Copyright $\odot 2020$ Li Wang et al. This is an open access article distributed under the Creative Commons Attribution License, which permits unrestricted use, distribution, and reproduction in any medium, provided the original work is properly cited.

\begin{abstract}
Objective. To evaluate the efficiency of sublingual immunotherapy with Dermatophagoides Farinae Drops in children with single and multiple respiratory allergic diseases. Methods. Seventy-one children with allergic respiratory diseases who had been treated with Dermatophagoides Farinae Drops for one year or more were divided into a single allergic group (12 cases) and multiple allergic group (59 cases). The rhinitis score, daytime and night symptom score of asthma, VAS score, drug score, pulmonary function, and FeNO level before and after treatment were evaluated and compared between the two groups. Results. The rhinitis score, night symptom score, VAS score, and drug score in the single allergic group after treatment were significantly lower than those before treatment $(p<0.05)$, but there was no significant difference in the daytime symptom score before and after treatment $(p>0.05)$. The rhinitis score, VAS score, and drug score in the multiple allergic group after treatment were significantly lower than those before treatment $(p<0.05)$, but there was no significant difference in the scores of daytime symptoms and nighttime symptoms before and after treatment $(p>0.05)$. In both the single allergic group and multiple allergic group, the pulmonary function indexes of the patients were significantly improved after treatment, and the FeNO after treatment was significantly lower than that before treatment $(p<0.05)$. There was no significant difference in scores, pulmonary function, and FeNO between the two groups $(p>0.05)$. Conclusion. Sublingual specific immunotherapy is effective in treating multiple and single allergic respiratory diseases in children.
\end{abstract}

\section{Introduction}

Respiratory allergic diseases are one of the most common diseases in pediatrics, including allergic asthma (AS), allergic rhinitis (AR), and cough variant asthma; among them, AS and $\mathrm{AR}$ are the most common respiratory allergic diseases in childhood [1]. In China, the prevalence of AR in children has increased from $9.1 \%$ in 2001 [2] to $15.4 \%$ in 2010 [3], and house dust mites have been documented to be the most prevalent allergens [4]. Symptomatic treatment is based on antihistamines and corticosteroids. Allergic asthma is characterized by chronic inflammation which results in recurrent attacks of cough, wheezing, sometimes chest tightness, and variable airflow obstruction $[5,6]$. It is a major public health problem affecting over 300 million people worldwide. According to the latest position papers, allergen specific immunotherapy has practically no controversies in the treatment of AR and allergic asthma [7]. Also, in the latest national guidelines for the diagnosis and treatment of $\mathrm{AR}$, it is suggested that allergen-specific immunotherapy should be used as first-line treatment for AR [8]. In the recent years, the incidence rate of allergic diseases in children has been increasing, which has caused great harm to children's physical and mental health, including a heavy economy burden on the family [9].

Dermatophagoides Farinae Drops, a sublingual immunotherapy drug, has been shown to be effective and safe in 
preschool and school-age patients with house dust mites(HDMs-) induced AR and adult patients with AA [10, 11]. Though the effectiveness of sublingual specific immunotherapy (SLIT) has been reported [12], there are relatively few data about the efficacy of sublingual Dermatophagoides Farinae Drops in the treatment of multiple and single respiratory allergic diseases in children. Therefore, this study evaluated the efficacy of sublingual Dermatophagoides Farinae Drops in children with multiple and single allergen respiratory allergic diseases.

\section{Methods}

2.1. Patients. From February 2016 to February 2018, 80 children diagnosed with asthma accompanied with rhinitis or not, who were treated with Dermatophagoides Farinae Drops, were selected. According to the skin prick test results, they were divided into two groups: (1) the single allergic group (20 cases): the children only showed positive for acarid dust or house dust mite. (2) The multiple allergy group (60 cases): 60 children were positive for, at least, one allergen besides mite.

The inclusion criteria were the following: (1) AS according to Guidelines for the Prevention and Treatment of AS in Children (2016) and Guidelines for Diagnosis and Treatment of AS in Children (Chongqing, 2010) [13, 14]; (2) the positive dust mite prick test with or without other allergens; and (3) nonacute asthma. The exclusion criteria were: (1) acute asthma (FEV1 less than $70 \%$ of predicted value); (2) severe allergic reaction.

2.2. Treatment. The sublingual Dermatophagoides Farinae Drops (S20060012) produced by Zhejiang Wowu Biotechnology Co., Ltd. were divided into 1-4 bottles, corresponding to the total protein concentration of $1,10,100$, and $333 \mu \mathrm{g} / \mathrm{ml}$, respectively.

The drug is kept under the tongue for 1-3 minutes before swallowing. The treatment includes an induction and a maintenance phase: the increasing period is one week for each bottle 1, 2, and 3 and from the first day to the seventh day of each week includes 1, 2, 3, 4, 6, 8, and 10 drops. During the maintenance period, Dermatophagoides Farinae Drops of bottles 4 were used and 3 drops/day were maintained until the end of the treatment. During the treatment of SLIT, other drugs for asthma and rhinitis were used according to the clinical symptoms of the children according to the guidelines $[6,15]$.

2.3. Pulmonary Function. The pulmonary function of children was evaluated by using the instrument from Jaeger (Master Screen, Jaeger, Germany). The measured parameters include forced vital capacity (FVC), forced expiratory volume in one second (FEV1), forced expiratory volume in one second (FEV1/FVC), maximum forced expiratory peak flow (PEF), forced expiratory volume in $25 \%, 50 \%$, and $75 \%$ of vital capacity (FEF25, FEF50, FEF75), and maximum mid expiratory flow (MMEF) [16].
2.4. Determination of Nitric Oxide. The exhaled nitric oxide was measured by using the exhaled nitric oxide tester (NIOX, MINO, Sweden). The level of FeNO was measured according to the standard measurement guide recommended by the American Thoracic Society/European Respiratory Society (ATS/ESR) [17].

2.5. Evaluation Indicators. The severity of rhinitis symptoms was evaluated with the "Four-point method" (Table 1) [18]. The symptom score of asthma includes the daytime symptom score, nighttime symptom score, and VAS score, which are evaluated according to the severity of symptoms and the impact on life (Table 2) [19].

The drug score was used to evaluate the application of drugs, which is mainly used to record the use of drugs pointing to symptoms in children to evaluate the clinical efficacy of immunotherapy. The "Three-steps" scoring method was used (Table 3) [14].

2.6. Statistical Analysis. SPSS 17.0 software was used to analyze the data. If the quantitative data obey the normal distribution, the mean \pm standard deviation $(\bar{X} \pm S)$ is used for description, and the $t$-test of two independent samples (Student's test) is used for group comparison. The median, upper, and lower quartiles (m, p25-p75) were used to describe the normal distribution of quantitative data, and the rank sum test of two independent samples was used to compare between groups (Mann-Whitney $U$-test). Qualitative data were described by percentage and compared by the chi square test. To the test level, $\alpha=0.05$ was used as the inspection standard.

\section{Results}

3.1. Basic Characteristics. Eighty children treated with Dermatophagoides Farinae Drops were included. In the single allergic group, 20 patients were enrolled. After one year's follow-up, 6 patients lost the follow-up and voluntarily withdrew. Two patients dropped out from treatment due to the acute asthma (FEV1 less than $70 \%$ of the predicted value). Finally, 12 patients completed the study, including 9 males (75.0\%) and 3 females (25.0\%). In the multiple allergy group, 60 people were enrolled, 1 person was lost to followup, and 59 people completed the study. There were 38 (64.4\%) males and 21 (35.6\%) females, respectively. There was no significant difference in gender composition between the two groups $(\chi 2=0.139, p=0.710)$. The age of the single sensitization group was $6.5 \pm 3.1$ years, and that of multiple sensitization group was $7.3 \pm 2.9$ years. There was no significant difference of age between the two groups $(t=0.856$, $p=0.395$ ).

Comparison of the rhinitis score, asthma score, VAS score, and drug score before and after treatment.

The rhinitis score, night symptom score, VAS score, and drug score after treatment in the single allergic group were significantly lower than those before treatment $(p<0.05)$, while there was no significant difference in the daytime 
TABLE 1: Allergic rhinitis symptom rating scale.

\begin{tabular}{lcccc}
\hline Grading & Sneeze $^{\mathrm{A}}$ & Shed tears $^{\mathrm{B}}$ & Antiques with history & Rhinopruritus \\
\hline 1 point & $3-5$ & $\leq 5$ & Inhale consciously & Interrupted \\
2 points & $6-10$ & $6-9$ & Intermittent or interactive & Allelopathy of ants, tolerable \\
3 points & $\geq 11$ & $\geq 10$ & Almost all day & Allelopathy of ants, intolerable \\
\hline
\end{tabular}

Note: ${ }^{A}$ the number of a consecutive sneezes; ${ }^{B}$ the number of daily nasal discharge.

TABLE 2: Evaluation of daytime and night symptoms of asthma.

\begin{tabular}{lcc}
\hline Grading & Daytime symptom score of asthma & Night symptom score of asthma \\
\hline 0 point & Asymptomatic & Asymptomatic \\
1 point & Few symptoms and short duration & Wake up once or early \\
2 points & Short symptoms $\geq 2$ times & Wake up twice, including early \\
3 points & Many times mild symptoms in day, little impact on life and work & Wake up many times \\
4 points & Many times serious symptoms in day, impact on life and work & Cannot sleep at night \\
5 points & The symptoms were so severe that the subjects could not work and live normally & \\
\hline
\end{tabular}

TABLE 3: Symptomatic drug rating scale.

\begin{tabular}{lc}
\hline Grading drug consumption scores & Drugs pointing symptom \\
\hline 1 point & Oral and/or local antihistamines, antileukotrienes, and bronchodilators \\
2 points & Nasal glucocorticoids/inhaled glucocorticoids \\
3 points & Oral glucocorticoids* \\
& Combination (glucocorticoids and $\beta 2$-receptor agonist) \\
\hline
\end{tabular}

${ }^{*}$ Oral glucocorticoids belong to the 3 points group.

symptom score before and after treatment $(p>0.05)$ (Table 4).

The rhinitis score, VAS score, and drug score after treatment in the multiple allergic group were significantly lower than those before treatment $(p<0.05)$, while there was no significant difference in the daytime symptom score and night symptom score before and after treatment $(p>0.05)$ (Table 5).

\subsection{Comparison of Pulmonary Function and FeNO before and} after Treatment. In both the single allergic group and multiple allergic group, the pulmonary function of the patients after treatment was significantly higher than that before treatment, while FeNO after treatment was significantly lower than that before treatment $(p<0.05)$ (Tables 6 and 7).

3.3. Comparison of the Two Groups. There was no significant difference of the scoring between the two groups $(p>0.05)$ (Table 8). There were also no significant differences of pulmonary function and FeNO between the two groups $(p>0.05)$ (Table 9).

\section{Discussion}

Allergen-specific immunotherapy (AIT) is considered as the only way to change the natural course of allergic diseases. The World Allergy Organization (WAO) recommends sublingual-specific immunotherapy (SLIT) as the initial and early treatment for allergic diseases and not when drugs are ineffective after the failure of drug treatment [20]. A meta-
TABLE 4: Comparison of scores of the single allergy group before and after treatment.

\begin{tabular}{lcccc}
\hline Variable & $\begin{array}{c}\text { Before } \\
\text { treatment }\end{array}$ & $\begin{array}{c}\text { After } \\
\text { treatment }\end{array}$ & $Z$ & $p$ \\
\hline Rhinitis score & $0.5(0.0,3.0)$ & $0.0(0.0,1.0)$ & 2.220 & 0.026 \\
$\begin{array}{l}\text { Daytime symptom } \\
\text { score }\end{array}$ & $0.0(0.0,0.5)$ & $0.0(0.0,0.0)$ & 1.633 & 0.102 \\
Night symptom & $2.5(0.0,4.0)$ & $0.0(0.0,0.0)$ & 2.375 & 0.018 \\
score & $5.0(3.0,5.5)$ & $0.5(0.0,2.0)$ & 2.940 & 0.003 \\
VAS score & $4.0(3.0,5.0)$ & $0.0(0.0,2.5)$ & 2.949 & 0.003 \\
Drug score & & & & \\
\hline
\end{tabular}

analysis confirmed the effectiveness of sublingual-specific immunotherapy in 3-18-year-old children with AS [21]. Another meta-analysis also confirmed that sublingual immunotherapy has a significant effect on children with AS and AR caused by dust mite allergy [22]. In this study, we have found that sublingual-specific immunotherapy of Dermatophagoides Farinae Drops is effective in treating multiple and single allergic respiratory diseases in children.

A large cross-sectional multicenter study in China found that more than $90 \%$ of allergic patients in China are allergic to two or more allergens, of which $83.7 \%$ are allergic to both dust mites and house dust mites [4]. Other studies also showed that the proportion of patients with multiple allergies was much higher than that of patients with single allergies [23]. Specific Dermatophagoides Farinae immunotherapy was effective in children with AS due to either single allergies or multiple allergies [24]. A retrospective study on 124 children with AR and AS who received SLIT treatment for 3 years showed that the sublingual 
TABLE 5: Comparison of scores in the multiple allergy group before and after treatment.

\begin{tabular}{|c|c|c|c|c|}
\hline Variable & Before treatment & After treatment & $Z$ & $p$ \\
\hline Rhinitis score & $0.0(0.0,2.0)$ & $0.0(0.0,1.0)$ & 4.520 & $<0.001$ \\
\hline Daytime symptom score & $0.0(0.0,1.0)$ & $0.0(0.0,1.0)$ & 1.448 & 0.148 \\
\hline Night symptom score & $1.0(0.0,3.0)$ & $0.0(0.0,4.0)$ & 1.024 & 0.306 \\
\hline VAS score & $4.0(2.0,6.0)$ & $1.0(0.0,2.0)$ & 6.052 & $<0.001$ \\
\hline Drug score & $4.0(3.0,4.5)$ & $0.0(0.0,2.0)$ & 6.571 & $<0.001$ \\
\hline
\end{tabular}

TABLE 6: Comparison of pulmonary function and FeNO in the single allergic group before and after treatment.

\begin{tabular}{|c|c|c|c|c|}
\hline Variable & Before treatment & After treatment & $t$ & $p$ \\
\hline FVC & $90.7 \pm 11.7$ & $103.9 \pm 12.4$ & 3.804 & 0.003 \\
\hline FEV1 & $84.1 \pm 11.0$ & $106.7 \pm 9.2$ & 8.093 & $<0.001$ \\
\hline $\mathrm{PEF}$ & $90.2 \pm 13.5$ & $108.3 \pm 4.0$ & 5.247 & $<0.001$ \\
\hline PEF75 & $52.2 \pm 20.1$ & $94.2 \pm 20.7$ & 4.998 & $<0.001$ \\
\hline PEF50 & $54.6 \pm 12.9$ & $94.8 \pm 18.9$ & 6.408 & $<0.001$ \\
\hline PEF25 & $58.6 \pm 17.6$ & $98.6 \pm 23.0$ & 4.602 & 0.001 \\
\hline MMEF & $52.8 \pm 12.9$ & $93.1 \pm 21.1$ & 5.080 & $<0.001$ \\
\hline $\mathrm{FeNO}$ & $39.5(25.5,89.0)$ & $18.5(14.0,57.5)$ & 3.061 & $0.002^{\mathrm{a}}$ \\
\hline
\end{tabular}

Note: (a) Mann-Whitney $U$-test of two independent samples.

TABLE 7: Comparison of pulmonary function and FeNO in the multiple allergic group before and after treatment.

\begin{tabular}{|c|c|c|c|c|}
\hline Variable & Before treatment & After treatment & $t$ & $p$ \\
\hline FVC & $89.2 \pm 11.4$ & $104.1 \pm 12.2$ & 9.761 & $<0.001$ \\
\hline FEV1 & $89.7 \pm 11.3$ & $105.6 \pm 13.5$ & 8.997 & $<0.001$ \\
\hline PEF & $92.3 \pm 13.1$ & $105.7 \pm 14.1$ & 6.764 & $<0.001$ \\
\hline PEF75 & $58.0 \pm 18.8$ & $89.5 \pm 24.9$ & 9.807 & $<0.001$ \\
\hline PEF50 & $70.1 \pm 14.8$ & $92.4 \pm 20.4$ & 8.827 & $<0.001$ \\
\hline PEF25 & $68.9 \pm 20.2$ & $97.2 \pm 19.8$ & 8.812 & $<0.001$ \\
\hline MMEF & $66.5 \pm 16.2$ & $90.9 \pm 22.8$ & 9.492 & $<0.001$ \\
\hline FeNO & $31.0(23.5,44.0)$ & $15.0(11.0,23.0)$ & 6.671 & $<0.001^{\mathrm{a}}$ \\
\hline
\end{tabular}

Note. (a) Mann-Whitney $U$-test of two independent samples.

TABLE 8: Comparison of scores between the single allergy group and multiple allergy group.

\begin{tabular}{lcrr}
\hline Variable & Single allergy group $(n=12)$ & Multiple allergy group $(n=59)$ & $Z$ \\
\hline Rhinitis score & $1(1,1)$ & $0(0,0)$ & 0.713 \\
Daytime symptom score & $0(0,0)$ & $0(0,0)$ & 0.476 \\
Night symptom score & $0(0,0)$ & $0(0,0)$ & 0.499 \\
VAS score & $2(1,3)$ & $0(0,2)$ & 0.792 \\
Drug score & $0(0,2)$ & $0(0,2)$ & 0.428 \\
\hline
\end{tabular}

TABLE 9: Comparison of pulmonary function and FeNO between single allergy groups and multiple allergy groups.

\begin{tabular}{lccc}
\hline Variable & Single allergy groups $(n=12)$ & Multiple allergy groups $(n=59)$ & $t$ \\
\hline FVC & $97.4 \pm 14.5$ & $97.9 \pm 11.9$ & 0.113 \\
FEV1 & $92.8 \pm 14.4$ & $97.4 \pm 12.2$ & 1.159 \\
PEF & $99.0(94.3,106.5)$ & $97.6(90.2,107.2)$ & 0.910 \\
PEF75 & $56.3 \pm 22.2$ & $60.6 \pm 20.2$ & 0.250 \\
PEF50 & $69.6 \pm 23.3$ & $78.6 \pm 18.1$ & $0.968^{\mathrm{a}}$ \\
PEF25 & $81.2 \pm 21.3$ & $92.7 \pm 18.0$ & 0.516 \\
MMEF & $65.8 \pm 22.0$ & $75.9 \pm 19.6$ & 0.139 \\
FeNO & $18.5(13.5,57.8)$ & $15.0(11.0,23.0)$ & 1.998 \\
\hline
\end{tabular}

Note. (a) Mann-Whitney $U$-test of two independent samples. 
Dermatophagoides Farinae had a similar effect on AR [25]. EAACI guidelines emphasize that sublingual desensitization with a single allergen were equally effective in patients with multiple allergies, which may be due to the cross reaction between different allergens and the inhibitory effect of cytokines on immune response [26].

There are a few studies evaluating the efficacy of sublingual Dermatophagoides Farinae in the treatment of single and multiple allergic respiratory diseases in children. A recent study showed that, after sublingual immunotherapy, the clinical indexes of children with both single and multiple allergies were significantly improved [27]. Another study also found that sublingual Dermatophagoides farinae can significantly improve the nasal symptoms of children with single dust mite allergy and dust mite combined with other allergies and reduce the use of drugs pointing to symptoms; moreover, the effect of Dermatophagoides farinae on children with single and multiple allergies was similar [28].

Our current study found that the rhinitis symptoms, asthma night symptoms, VAS scores, and drug scores were significantly improved in the single allergic group, but the daytime symptoms were not significantly improved after treatment. Rhinitis symptoms, VAS scores, and drug scores were significantly improved in the multiple allergy group, but the daytime symptoms and night symptoms were not significantly improved before and after treatment. Our study also confirmed that the efficacy of sublingual Dermatophagoides farinae drops in the treatment of respiratory allergic diseases is equivalent in children with single and multiple allergies, which was in accordance with a previous study [27].

The measurement of pulmonary function has been one of the important bases for the diagnosis and evaluation of asthma control. FeNO is also one of the most valuable markers reflecting allergic airway inflammation [29]. Therefore, pulmonary function and FeNO can be used as the evaluation indexes of SLIT in the treatment of respiratory allergic diseases. This study showed that the pulmonary function indexes and FeNO levels were significantly improved in both single and multiple allergic groups. It was found that sublingual-specific immunotherapy can improve pulmonary function (PEF, FEV1\%) and FeNO in children with AS and AR [30]. SLIT treatment can significantly improve the symptoms, reduce the level of FeNO, reduce airway inflammation, and improve the ventilation index of large and small airways in children with AS and AR [31].

A previous study showed that the sublingual immunotherapy of house dust mite could significantly improve the airflow obstruction (FEV1), significantly reduce the level of FeNO, and reduce the eosinophilic inflammation of the airway in patients with AS and AR [32]. Another study also showed that SLIT could significantly improve the symptoms, reduce the long-term use of drugs, and improve FEV1 [33], which was also in accordance with our results. However, one study including 26 children with AS and AR, treated with SLIT and followed up for 3 years, had no significant improvement in pulmonary function [34]. A retrospective study of 124 children with AS and AR treated with SLIT for 3 years showed that there was no significant difference in the improvement of lung function (PEF, FEV1) of children with single or multiple allergies by SLIT [25]. Therefore, further study with lager sample size was needed to confirm the conclusion. There was no study investigating the difference in the improvement of small airways (FEF25-75, fef75/fef25) and FeNO levels in children with single or multiple allergy treated with SLIT. In this study, it was found that the treatment of sublingual Dermatophagoides Farinae drops could significantly improve the small airway index (FEF2575 , fef75/fef25) and the level of FeNO in the two groups.

In conclusion, our study found that sublingual-specific immunotherapy was effective in treating multiple and single allergic respiratory diseases in children, which further confirmed the clinical use of Dermatophagoides Farinae Drops in respiratory allergic diseases.

\section{Data Availability}

The authors agree to share the data and materials of this paper.

\section{Conflicts of Interest}

The authors declare that they have no conflicts of interest to report regarding the present study.

\section{Acknowledgments}

The study was supported by the Health Research Project of Sichuan Province (17PJ277).

\section{References}

[1] L. Xiang, J. Zhao, Y. Bao et al., "Expert consensus on mite specific immunotherapy for allergic airway diseases in children," Chinese Journal of Practical Pediatrics, vol. 33, no. 16, pp. 1215-1223, 2018.

[2] G. W. K. Wong, D. S. C. Hui, H. H. Chan et al., "Prevalence of respiratory and atopic disorders in Chinese schoolchildren," Clinical \& Experimental Allergy, vol. 31, no. 8, pp. 1225-1231, 2001.

[3] J. Zhao, J. Bai, K. Shen et al., "Self-reported prevalence of childhood allergic diseases in three cities of China: a multicenter study," BMC Public Health, vol. 10, p. 551, 2010.

[4] J. Li, B. Sun, Y. Huang et al., "A multicentre study assessing the prevalence of sensitizations in patients with asthma and/or rhinitis in China," Allergy, vol. 64, no. 7, pp. 1083-1092, 2009.

[5] A. Yukselen and S. G. Kendirli, "Role of immunotherapy in the treatment of allergic asthma," World Journal of Clinical Cases, vol. 2, no. 12, p. 859, 2014.

[6] A. D. Hogan and J. A. Bernstein, "GINA updated 2019," Annals of Allergy, Asthma \& Immunology, vol. 124, no. 4, pp. 311-313, 2020.

[7] M. Jutel, I. Agache, S. Bonini et al., "International consensus on allergy immunotherapy," Journal of Allergy and Clinical Immunology, vol. 136, no. 3, pp. 556-568, 2015.

[8] Subspecialty Group of Rhinology, "Editorial board of chi-nese journal of otorhinolaryngology head and neck surgery; subspecialty group of rhinology, society of otorhinolaryn-gology head and neck surgery. Chinese medical association. Chinese guidelines for diagnosis and treatment of allergic rhinitis," 
Chinese Journal of Otorhinolaryngology Head and Neck, vol. 51, pp. 6-24, 2016.

[9] S. Halken, D. Larenas-Linnemann, G. Roberts et al., "EAACI guidelines on allergen immunotherapy: prevention of allergy," Pediatric Allergy and Immunology, vol. 28, no. 8, pp. 728-745, 2017.

[10] L.-X. Tang, X.-J. Yang, P.-P. Wang et al., "Efficacy and safety of sublingual immunotherapy with dermatophagoides farinae drops in pre-school and school-age children with allergic rhinitis," Allergologia et Immunopathologia, vol. 46, no. 2, pp. 107-111, 2018.

[11] C. Zhong, W. Yang, Y. Li et al., "Clinical evaluation for sublingual immunotherapy with dermatophagoides farinae drops in adult patients with allergic asthma," Irish Journal of Medical Science (1971), vol. 187, no. 2, pp. 441-446, 2018.

[12] G. Roberts, O. Pfaar, C. A. Akdis et al., "EAACI guidelines on allergen immunotherapy: allergic rhinoconjunctivitis," $\mathrm{Al}$ lergy, vol. 73, no. 4, pp. 765-798, 2018.

[13] Respiratory group of Pediatric Society of Chinese Medical Association and Editorial Board of Chinese Journal of Pediatrics, "Guidelines for the diagnosis and prevention of bronchial asthma in children (2016 Edition)," Chinese Journal of Pediatrics, vol. 054, no. 3, pp. 167-181, 2016.

[14] Chinese Journal of Otorhinolaryngology Head and Neck Surgery Editorial Committee, "Chinese Medical Association otorhinolaryngology head and neck surgery credit association, pediatric group, Editorial Committee of Chinese Journal of Pediatrics. Expert consensus on the diagnosis and treatment of children's allergic rhinitis (2010, Chongqing)," Chinese Journal of Pediatrics, vol. 49, no. 2, pp. 116-117, 2011.

[15] M. D. Seidman, R. K. Gurgel, S. Y. Lin et al., "Clinical practice guideline," Otolaryngology-Head and Neck Surgery, vol. 152, no. 1, pp. S1-S43, 2015.

[16] "Pulmonary function group, respiratory branch, Chinese medical association. guidelines for pulmonary function examination (part II) - spirometry examination," Chinese Journal of Tuberculosis and Respiration, vol. 37, no. 7, pp. 481-486, 2014.

[17] R. A. Dweik, P. B. Boggs, S. C. Erzurum et al., "An official ATS clinical practice guideline: interpretation of exhaled nitric oxide levels (FENO) for clinical applications," American Journal of Respiratory and Critical Care Medicine, vol. 184, no. 5, pp. 602-615, 2011.

[18] O. Pfaar, P. Demoly, R. Gerth van Wijk et al., "Recommendations for the standardization of clinical outcomes used in allergen immunotherapy trials for allergic rhinoconjunctivitis: an EAACI Position Paper," Allergy, vol. 69, no. 7, pp. 854-867, 2014.

[19] P. C. Potter, "Update on sublingual immunotherapy," Annals of Allergy, Asthma \& Immunology, vol. 96, no. 2, pp. S22-S25, 2006.

[20] G. W. Canonica, L. Cox, R. Pawankar et al., "Sublingual immunotherapy: world allergy organization position paper 2013 update," World Allergy Organization Journal, vol. 7, no. 1, p. 6, 2014.

[21] M. Penagos, E. Compalati, F. Tarantini et al., "Efficacy of sublingual immunotherapy in the treatment of allergic rhinitis in pediatric patients 3 to 18 years of age: a meta-analysis of randomized, placebo-controlled, double-blind trials," Annals of Allergy, Asthma \& Immunology, vol. 97, no. 2, pp. 141-148, 2006.

[22] L. Cheng and W.-C. Zhou, "Sublingual immunotherapy of house dust mite respiratory allergy in China," Allergologia et Immunopathologia, vol. 47, no. 1, pp. 85-89, 2019.
[23] L. S. Cox, D. L. Linnemann, H. Nolte, D. Weldon, I. Finegold, and H. S. Nelson, "Sublingual immunotherapy: a comprehensive review," Journal of Allergy and Clinical Immunology, vol. 117, no. 5, pp. 1021-1035, 2006.

[24] L. Cui, J. Li, Y. Li, and Z. Xia, "Long-term efficacy of sublingual mite immunotherapy in monosensitized and polysensitized children with allergic rhinitis: a 7-year prospective study," International Archives of Allergy and Immunology, vol. 180, no. 2, pp. 144-149, 2019.

[25] Q. Xing, S. Chen, B. Chen et al., "Evaluation of the efficacy of sublingual dust mite drops in the treatment of single and multiple sensitized allergic rhinitis with asthma in children," Journal of Otolaryngology Head and Neck Surgery, vol. 031, no. 3, pp. 204-208, 2017.

[26] A. N. Pepper, M. A. Calderón, and T. B. Casale, "Sublingual immunotherapy for the polyallergic patient," The Journal of Allergy and Clinical Immunology: In Practice, vol. 5, no. 1, pp. 41-45, 2017.

[27] P. Li, Q. Li, Z. Huang, W. Chen, Y. Lu, and M. Tian, "Efficacy and safety of house dust mite sublingual immunotherapy in monosensitized and polysensitized children with respiratory allergic diseases," International Forum of Allergy \& Rhinology, vol. 4, no. 10, pp. 796-801, 2014.

[28] J. Wang, C. Chang, M. Wei et al., "Observation on the efficacy of sublingual dust mite drops in the treatment of allergic rhinitis in children with single or multiple allergens," Journal of Clinical Otorhinolaryngology Head and Neck Surgery, vol. 032, no. 21, pp. 1646-1650, 2018.

[29] F. C. L. Hoyte, L. M. Gross, and R. K. Katial, "Exhaled nitric oxide," Immunology and Allergy Clinics of North America, vol. 38, no. 4, pp. 573-585, 2018.

[30] Q. Huang, X. Lin, X. Wei et al., "Efficacy analysis of specific immunotherapy in patients with allergic asthma and allergic rhinitis," Chinese Journal of Clinicians, vol. 47, no. 3, pp. 79-82, 2019.

[31] X. Li, "Clinical efficacy of slit in the treatment of children with asthma and allergic rhinitis," 2019.

[32] M. Hoshino, K. Akitsu, and K. Kubota, "Effect of sublingual immunotherapy on airway inflammation and airway wall thickness in allergic asthma," The Journal of Allergy and Clinical Immunology: In Practice, vol. 7, no. 8, pp. 2804-2811, 2019.

[33] S. Y. Lin, A. Azar, C. Suarez-Cuervo et al., "Role of sublingual immunotherapy in the treatment of asthma: an updated systematic review," International Forum of Allergy \& Rhinology, vol. 8, no. 9, pp. 982-992, 2018.

[34] H. Huang, X. Liu, X. Hou et al., "The effect of dust mite allergen immunotherapy combined with drug therapy on the clinical efficacy and airway hyperresponsiveness of children with asthma and allergic rhinitis," Journal of Capital Medical University, vol. 37, no. 5, pp. 568-573, 2016. 